\title{
We can change practice-can we also change culture?
}

\author{
Fiona Godlee editor in chief
}

The BMJ

Guidelines can be hard to follow, as Helen Salisbury finds this week (doi:10.1136/bmj.154). They can also be slow to update in response to new evidence. This is something our Rapid Recommendations series aims to change. The Rapid Recommendations editors leap into action when they spot research that should change practice, in this case a randomised trial published last August in the New England Journal of Medicine. This found that among people who have had a mild stroke or high risk transient ischaemic attack, dual antiplatelet therapy with aspirin and clopidogrel reduced the risk of recurrent stroke when compared with aspirin alone.

The Rapid Recommendations meta-analysis (doi:10.1136/bmj. k5108) incorporates two other high quality randomised trials and confirms the benefit of dual therapy if given within 24 hours of the onset of symptoms. The Rapid Recommendations expert panel, including four patients and carers, concludes that treatment for 10-21 days gives the best balance between benefit and harm (mainly gastrointestinal bleeding), after which patients should continue with aspirin alone (doi:10.1136/bmj.k5130).

Less clear is how best to manage a ruptured Achilles tendon. Operative and non-operative treatment offer different pros and cons, say Yassine Ochen and colleagues in their systematic review and meta-analysis (doi:10.1136/bmj.k5120). The accompanying editorial (doi:10.1136/bmj.k5344) suggests that non-operative management is cheaper and avoids surgical complications, but surgery may give the patient greater confidence and a faster return to activity. The authors recommend a personalised approach and fully informed shared decision making.

Elsewhere this week we hear a range of responses to the UK government's long term plan for the NHS (doi:10.1136/bmj.180, doi:10.1136/bmj.185, doi:10.1136/bmj.187, doi:10.1136/bmj. 1107). We also learn that burnout, such a sadly familiar term in healthcare, was first described in air traffic controllers. Rajvinder Samra explains that evidence from aviation challenges the emphasis that medical education places on developing personal resilience (doi:10.1136/bmj.k5268). Instead, a key factor in burnout is the increasing complexity of the work, thus we need to look at human factors and the effect of the working environment. Aviation teams are trained together in situational awareness, decision making, communication, and problem solving. They are also trained in how to deal with the stress of critical incidents. Much of this is transferable to medicine, says Samra, and could help to improve the culture of healthcare.

Samra doesn't mention the role of safe spaces to debrief and re-energise. But Abi Rimmer does, in her article on why we still need doctors' messes (doi:10.1136/bmj.k5367). She reports on two hospitals where messes have been transformed by individual doctors' hard work and imagination. If you have a story about your doctors' mess we'd like to hear from you. Send a rapid response to this article or tweet using the hashtag \#GiveUsABreak. 